\title{
Performance Evaluation of Filters for Enhancement of Images in Different Application Areas
}

\author{
Yogendra Kumar Jain ${ }^{1}$,GARIMA SILAKARI ${ }^{2}$ \\ ${ }^{1}$ H.O.D. ,Department of Computer Science and Engineering, S.A.T.I Vidisha, India, \\ ${ }^{2}$ M.Tech Scholar, Department of Computer Science and Engineering , S.A.T.I Vidisha, India,
}

\begin{abstract}
Contrast stretching is a method to enhance the image details. Histogram equalization is one of the most common spatial domain technique used for contrast enhancement. While stretching the contrast of an image, it results in the degradation of brightness of the image. To overcome this hurdle a method known as BiHistogram equalization was introduced to preserve the brightness level of the output image. As the contrast enhancement enhances the image, it also enhances the noise in the signal that causes the blurrness in the output image. The proposed BHEGF method reduces this drawback and provides more accurate and realistic results. In this paper we use four different types of filters i.e. median filter, gaussian filter, average filter \& motion filter and various parameters such as processing time, MSE, brightness count and PSNR are used for performance evaluation.
\end{abstract}

Keywords: Bi- Histogram Equalization, Tri- histogram equalization ,BHEGF , Contrast enhancement, Gaussian filter, brightness preserving, Spatial and frequency domain.

\section{Introduction}

The main purpose of image enhancement is to enhance or improve the quality of image with the minimum amount of MSE. Various enhancement techniques are used for this purpose both in spatial domain and frequency domain. This paper emphasizes mainly on spatial domain technique of image enhancement. The histogram equalization is the most commonly used technique for contrast enhancement and brightness preservation of the image. There are various techniques used to equalizes the given image's histogram such as BHE, DSHE, AHE, CHE, MHE, MMBEBHE, RMSHE. In all these technique the image have to first decomposed and then the histogram of each part generated from the decomposition is equalized. That is also known as the segmentation of an image into various sub-images.

Segmentation of the image can be done in various ways such as amplitude thresholding, component labeling, boundary based approaches, region based approaches, template matching, texture based segmentation. In this paper, the image is segmentized on the bases of its component levels. Then the resultant histograms of the sub-images are equalized, while equalizing the histograms the impulse noises present in the images are also get enhanced along with the enhancement of the image [4] . Hence to overcome this drawback due to noise in the satellite imaging, this paper proposed the use of the Gaussian filter to filter out the noise from the enhanced image. The use of Gaussian filter shell reduced the MSE to a larger extent from the enhanced image .

\subsection{Image Enhancement}

Image enhancement is a process of improving the quality of image by improving its features. The techniques used for image enhancement can be used to improve the image's contrast and brightness characteristics and reduces its noise contents or sharpen its details they can be classified as subjective enhancement and objective enhancement [9]. Subjective enhancement techniques may be repeatedly applied in various forms until the observer feels that the image yields the details necessary for particular application.

On the other hand objective image enhancement corrects an image for known degradation. This enhancement is not repeatedly applied but it is applied only once based on the measurement taken from the system.

Image enhancement can also be categorized into two main and broad categories. They are as follows:

\subsubsection{Spatial Domain Technique}

Spatial domain refers to the Image Plane itself and approaches in this categories are based on direct manipulation of pixels in an image. Histogram equalization techniques are one of the spatial domain image enhancement technique, which has form the basis in this paper. This technique refers to the aggregate of the pixels composing an image. This process will be denoted by the expression given below :

$$
\mathrm{A}(\mathrm{x}, \mathrm{y})=\mathrm{T}(\mathrm{f}(\mathrm{x}, \mathrm{y}))
$$

Where $\mathrm{f}(\mathrm{x}, \mathrm{y})$ is input image, $\mathrm{g}(\mathrm{x}, \mathrm{y})$ is processed image and $\mathrm{T}$ is an operator on $\mathrm{f}$, given $\mathrm{g}(\mathrm{x}, \mathrm{y})=\mathrm{A}(\mathrm{x}$, y). 


\subsubsection{Frequency Domain Technique :}

frequency domain processing techniques are based on modifying the Fourier transform of an image [4].

\section{2 histogram equalization}

Histogram equalization is a technique to obtain a uniform histogram for the output image. It flattens the histogram and stretches the dynamic range of gray levels or in other words histogram equalization maps the input image's intensity values over the range ( 0 to 255 ) so that the histogram of the resulting image will have an approximately uniform distribution. This technique is used for contrast stretching and certain modification in this technique can make it useful for preserving the brightness of the image. Due to this reason, the histogram equalization has been found to be a powerful technique for image enhancement.

We have to generate random variables for histograms to be equalized, Consider an image pixel value $u>=0$ to be a random variable which has a continuous Probability Density Function and cumulative probability distribution.

Consider an image $I=\{X(i, j)\}$ is an image with $L$ discrete gray levels $\left\{X_{0}, X_{1}, X_{2}, \ldots \ldots, X_{L-1}\right\}$. The histogram of $I[X]$ is denoted by H[I $]=\left\{n_{0}, n_{1}, n_{2}, \ldots \ldots ., n_{k}, \ldots \ldots \ldots, n_{L-1}\right\}$ where $n_{k}$ is the number of pixels whose gray level is $\mathrm{X}_{\mathrm{k}}$. The mean brightness of the image $\mathrm{I}[\mathrm{X}]$ will be denoted as $\mathrm{I}_{\mathrm{MB}}$. The middle gray level of the image I $[\mathrm{X}]$ i.e. $\left(\mathrm{X}_{0}+\mathrm{X}_{\mathrm{L}-1}\right) / 2$ will be denoted as $\mathrm{X}_{\mathrm{G}}$. Consider the input image as $\mathrm{I}[\mathrm{X}]$. The Probability Density Function (PDF) of the image based on the histogram $\mathrm{H}[\mathrm{X}]$ can be defined as

$$
\mathrm{P}(\mathrm{K})=\mathrm{K} / \mathrm{N}=\mathrm{K} /\left(\mathrm{n}_{0}+\mathrm{n}_{1}+\mathrm{n}_{2}+\ldots \ldots . .+\mathrm{n}_{\mathrm{L}-1}\right) \text { for } \mathrm{K}=0,1,2,3 \ldots \ldots \ldots ., \mathrm{L}-1 .
$$

Here $\mathrm{N}$ is the total number of pixels in the image. Now the Cumulative Distribution Function (CDF) is calculated based on PDF is as follows :

$$
\mathrm{C}(\mathrm{K})=\sum_{\mathrm{j}=0}^{\mathrm{K}} \mathrm{P}(\mathrm{j}) \text { for } \mathrm{K}=0,1,2,3, \ldots \ldots ., \mathrm{L}-1
$$

Based on $\mathrm{CDF}$, Histogram equalization now maps an input gray level $\mathrm{X}_{\mathrm{K}}$ into an output gray level $\mathrm{f}(\mathrm{K})$, where $\mathrm{f}(\mathrm{K})$ commonly called a level transformation function is defined as

$$
\mathrm{C}(\mathrm{K})=\mathrm{X}_{0}+\left(\mathrm{X}_{\mathrm{L}-1}-\mathrm{X}_{0}\right) \cdot \mathrm{C}(\mathrm{K})[10]
$$

\section{Previous Work}

This section describes some previously done work based on the Histogram Equalization method with the purpose of brightness preservation. Firstly we are going to describe the various ways used to equalized the histogram of the images. The very first technique is Classical Histogram Equalization which forms the base of other spatial domain techniques. For the much better results we have to segmentize the given image into subimages. So in later part of this section we have described the concept of segmentation also. The segmentation of image is common in the various histogram equalization techniques but the difference lies in the way the segmentation is done in various techniques, one technique simply divides the image into two sub-images using mean gray level, other uses recursive segmentation, another uses the equal area value to segment the image . The various ways to equalizes the Histogram of the image and maintains the contrast and brightness of the image are as follows :

2.1 Classical Histogram Equalization (CHE): This technique emphasizes on the basic concept of Histogram Equalization which uniformly distribute the histogram of image over the entire range of gray levels increasing the contrast of the image . Despite of the advantages offered by the CHE it can introduce a significant change in the image brightness i.e. its mean gray level [3]. It is because of the uniform distribution specification of the output image to the middle gray level. This change in brightness is not desirable when applying the CHE technique.

2.2 Adaptive Histogram Equalization (AHE): Since the simple histogram equalization changes the brightness of the image significantly, and makes the images saturated wit bright or dark intensity values, therefore, the adaptive histogram equalization technique divide the histogram into several sub sections and equalizes them independently.

2.3 Brightness Preserving Histogram Equalization(BBHE):In this technique the histogram of image is first decomposed into two sub-histogram by using the mean gray level. Then the classical histogram equalization is applied to the histogram of the sub images independently.

2.4 Dualistic Sub-Image Histogram Equalization (DSIHE): Using the basic idea of BBHE of decomposing the original image into two sub-images and then equalize the histogram of the sub-images separately, [ ] proposed the so called equal area dualistic sub image histogram equalization. In this method decomposition is done on the bases of maximization of the entropy of the output image[ ]. For this the image is decomposed into two sub images one is dark and other is bright based on the property that the sub images will have same amount of pixels.

2.5 Minimum Mean Brightness Error Bi-Histogram Equalization (MMBEBHE) : This method provides a technique in which the decomposition can be done on the bases of the determination of a threshold level, 
let it be L1 on the bases of which the input image I can be decomposed into two sub images as I[0,L1] and $\mathrm{I}[\mathrm{L} 1+1, \mathrm{~N}]$ so that the minimum brightness difference $\mathrm{b} / \mathrm{w}$ the input image and the output image is achieved. Once the input image is decomposed by the threshold level L1, each of the two sub images $\mathrm{I}[0, \mathrm{~L} 1]$ and $\mathrm{I}[\mathrm{L} 1+1, \mathrm{~N}]$ has its histogram equalized by the CHE process generating the output image . Here in this paper we have proposed a scheme which is a modification of MMBEBHE and multi histogram equalization. We have introduce a technique uses various filters to reduce the noise and improve the overall quality of the image.

2.6 Recursive Mean - Separate Histogram Equalization(RMSHE): This method is based on BBHE but the difference in $\mathrm{b} / \mathrm{w}$ both the methods is that in this method instead of decomposing the image only once here to perform image decomposition recursively, up to a scale $r$, generating $2^{r}$ sub images. After it each of these sub images $\mathrm{I}^{\mathrm{r}}[\mathrm{I} 1$, I2] is independently enhanced using the CHE method.

\section{Proposed Technique}

In our proposed scheme the input image is decomposed into sub-images by using the threshold gray level which is selected on the basis of image mean gray level. Then apply the histogram equalization method on each of the sub - image's histogram to map the values into the new dynamic range . now normalizes the brightness of the image. There is an enhancement also in the noise of the image, so for the reduction of the noise and to obtain the more accurate information extraction from the satellite images which is most important for application based specific task done accurately such as in space research organization, weather forecasting centre etc. are the application areas related to satellite image information extraction

In this paper, the comparative studies has done among the performance of the four most prominently used filters along with contrast enhancement and brightness preservation. The filters used in this paper are Median filter, Gaussian filter, Average filter and Motion filter along with the multi - histogram equalization technique .

\subsection{Median Filter}

A median filter finds the median of a number of elements at its input. The median of a group containing an odd number of elements is defined as the middle element when all the elements of the group is sorted . In the standard the median filtering applications a window of size $\mathrm{W}$, where $\mathrm{W}$ is odd is moved along the sampled values of the signal or image. The dimensions of the filter mask must be odd [4].

\subsection{Gaussian Filter}

In Gaussian filtering the weights are computed according to the Gaussian function i.e. $g(i, j)=c . e^{\left(1^{2}+j^{2}\right) /}$. Gaussian filtering is very effective in removing Gaussian noise \& the weight give higher significance to pixels near the edges (removes egde blurring). They are linear low pass filters . Large filters can be implemented using small 1D small filters also they are rotationally symmetric and computationally more efficient .

\subsection{Average Filter}

Average filtering is also a sort of low pass filtering in which average of pixel values are taken to reduce the effect of noise in the image . suppose we have $n$ measurements of pixel $\mathrm{x}$. then the standard deviation of this measured pixel $\mathrm{x}$ can be estimated as :

$$
\begin{aligned}
& \mathrm{S}=\sqrt{ }\left(\sum_{\mathrm{i}=1}^{\mathrm{x}}(\mathrm{Xi}-\mathrm{X})^{2}\right) /(\mathrm{n}-1) \\
& \text { Where } \mathrm{X} \text { is the mean or average of } \mathrm{n} \text { measurements calculated as : } \\
& \quad \mathrm{X}=1 / \mathrm{n}\left(\sum_{\mathrm{i}=1}^{\mathrm{x}} \overline{\mathrm{X}} \mathrm{i}\right)
\end{aligned}
$$

The magnitude of $\mathrm{S}$ is clearly dependent on the measurements, $\mathrm{Xi}$, which in practice is bounded, that is it has lower and upper limits. However $\mathrm{S}$ is also dependent on the number of measurements made i.e. the number $\mathrm{n}$, From such calculations it is deduced that the larger the $\mathrm{n}$ is, the smaller the $\mathrm{S}$ becomes . In other words, given a noisy but bounded measurement sequence, we can take a large number of readings of pixel and use its average to give a better estimate of its true value .

\subsection{Motion Filter}

As the result of motion between the camera and the object of interest, adjacent point in the image plane are exposed to the same point in the object plane during the exposure time. This causes the blurriness in the image and it is termed as motion blurred image. Thus motion filter is used to reduce such blurriness from the image with the help of motion function PSF .[1].

Here the proposed method can be summarized as follows :

- $\quad$ Decomposing the image into sub images using its threshold value.

- Stretches the contrast and preserve the brightness of the image by using histogram equalization which flattens and stretches dynamic range of histogram of the image .

- $\quad$ Filtering process can be done using median filter on the stretched image. 
- Now apply the Gaussian filter to reduce the Gaussian noise .

- $\quad$ Further apply average filter to the stretched image .

- Lastly apply motion filter to the stretched image .

- At the last step compare the results and brightness measurements come from the above filters and multi histogram equalization technique on the basis of brightness count, MSE count and PSNR values .

\section{Results}

Thus this paper conclude that the Gaussian filter gives much better results in comparison to all other filters in the case of satellite images when applied with BBHE. In other words the MSE count is much lesser with Gaussian filter compared to other one and it also gives a very good PSNR count. This will be very good results with both gray scale and color images. This technique can be extended to 3D images also and other application areas. Following are some results.

\subsection{Lung-Vessel}

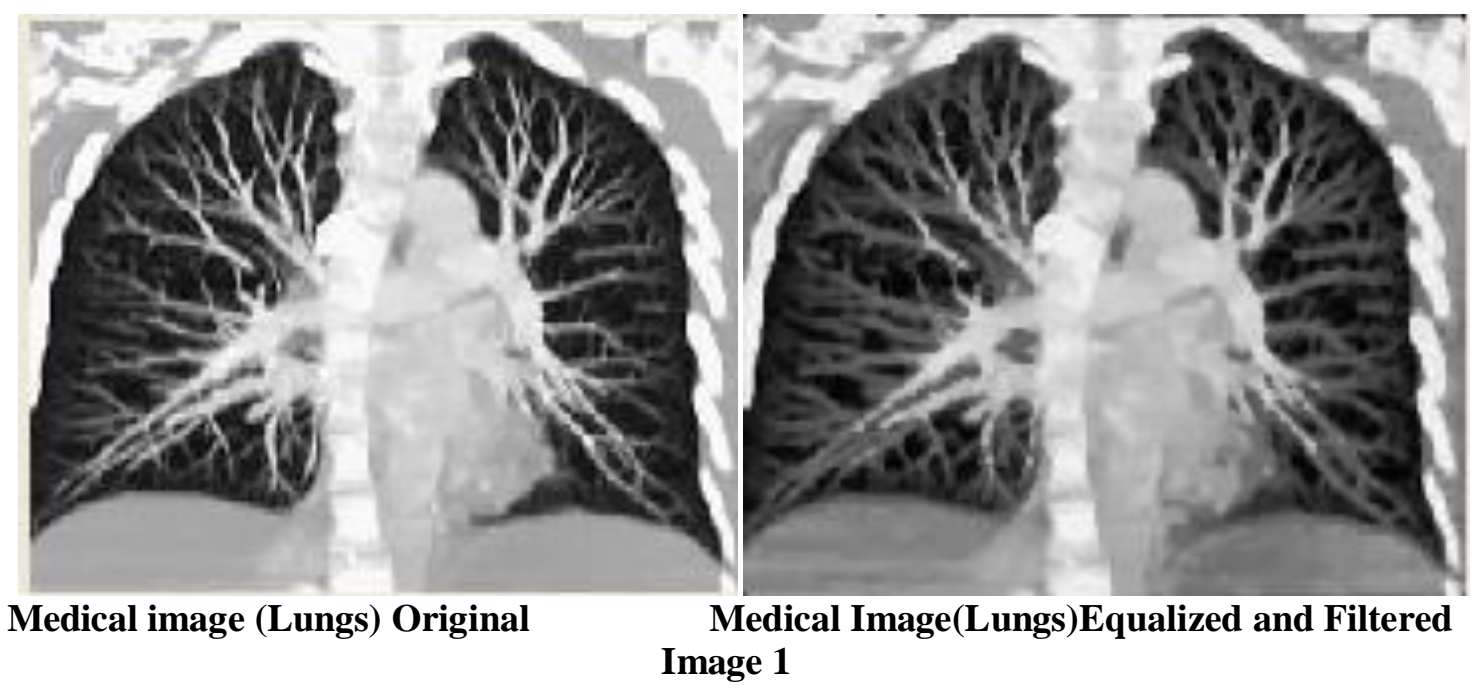

\section{Histograms for Medical image (Lungs)}
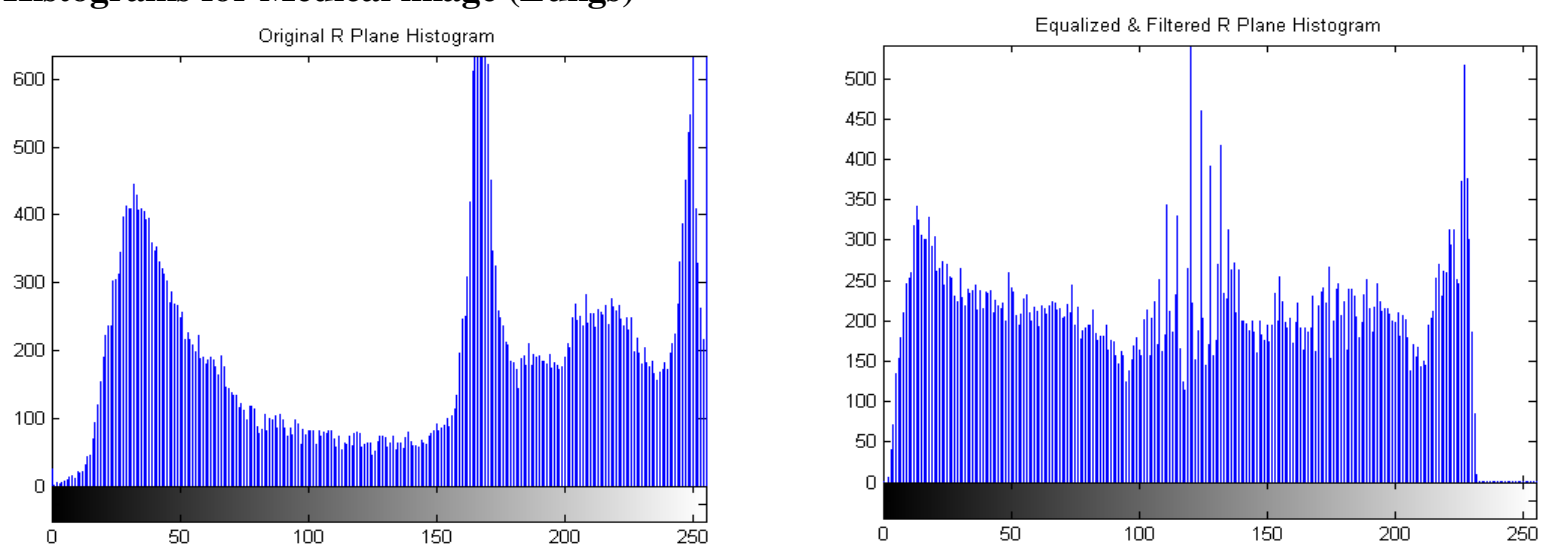

Fig. 1(A) R-component of original image

Fig. 1(B) R-component of equalized and filtered image. 


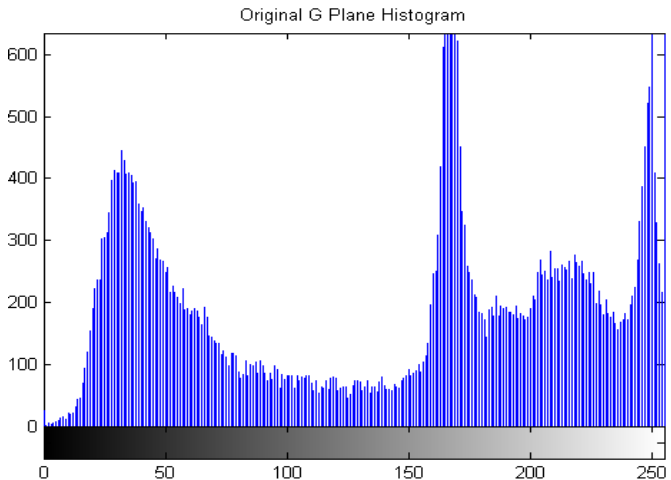

Fig. 1(C) G-component of original image

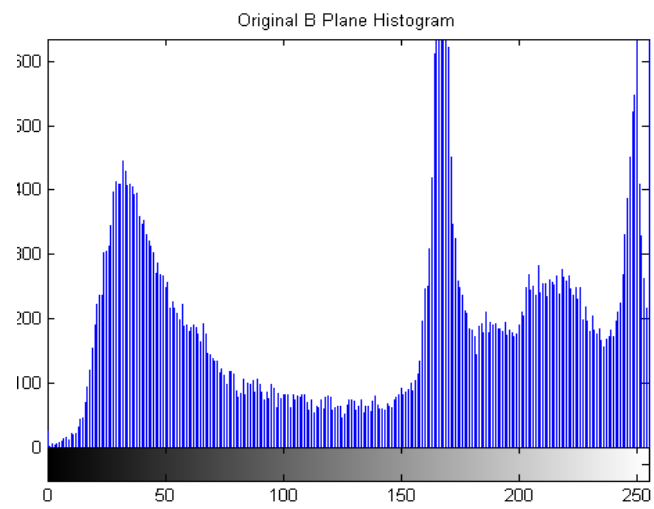

Fig. 1(E) B-component of original image

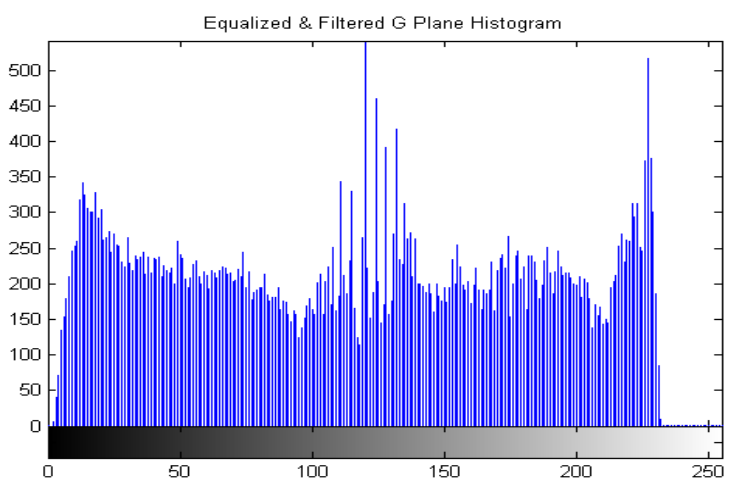

Fig. 1(D) G-component of equalized and Filtered image .

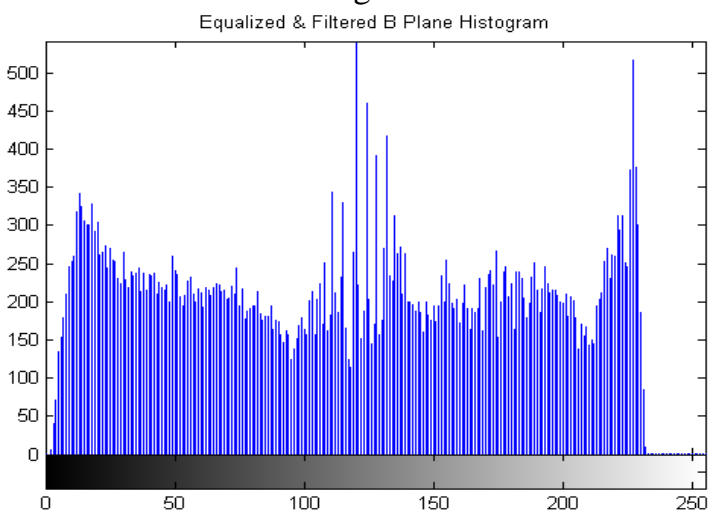

Fig. 1(E) B-component of equalized and filtered image .

Fig. 1

Fig. 1 . Histograms of Lungs CT Images from medical imaging regarding R, G, B color components of original and equalized and filtered by using Gaussian filter .

\subsection{Oilpill}

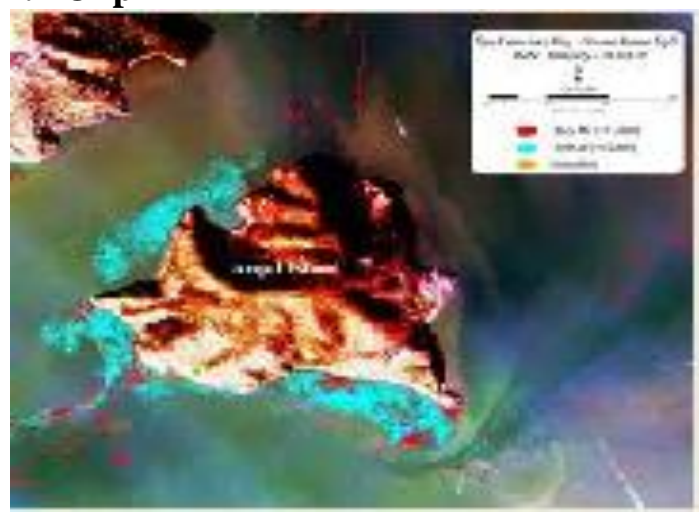

Oilpill Original

\section{Histograms of Colaba's image :}

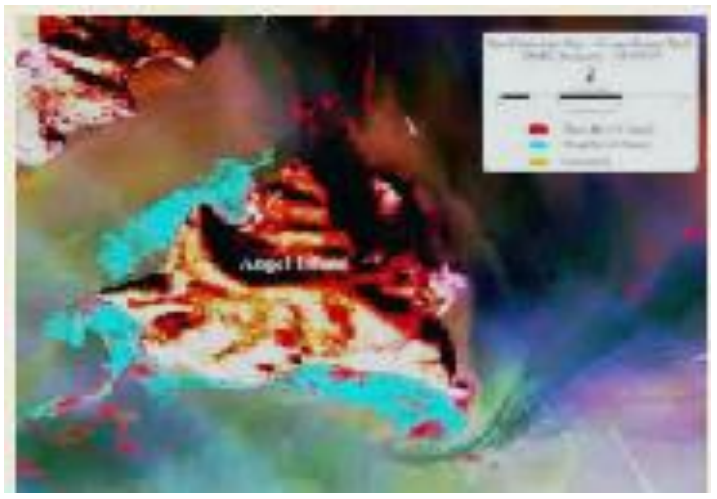

Oilpill equalized and filtered

\section{Image 2}




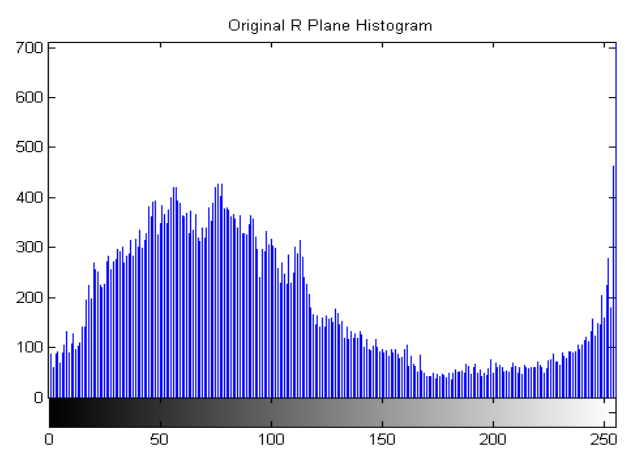

Fig. 2(A) R-component of original image .

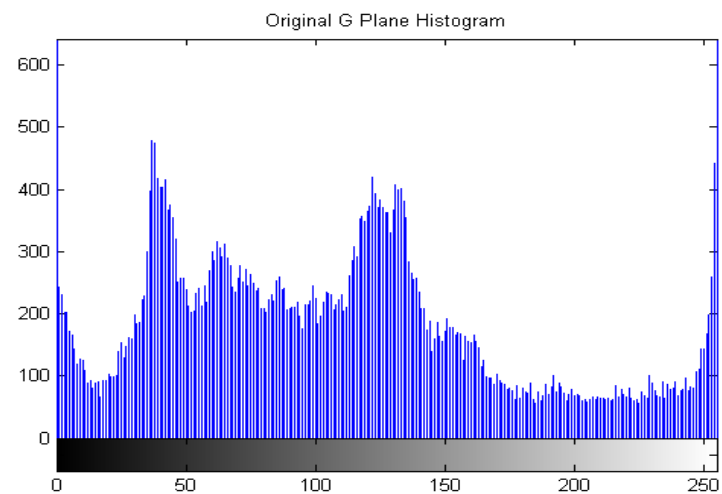

Fig. 2(C) G-component of original image .

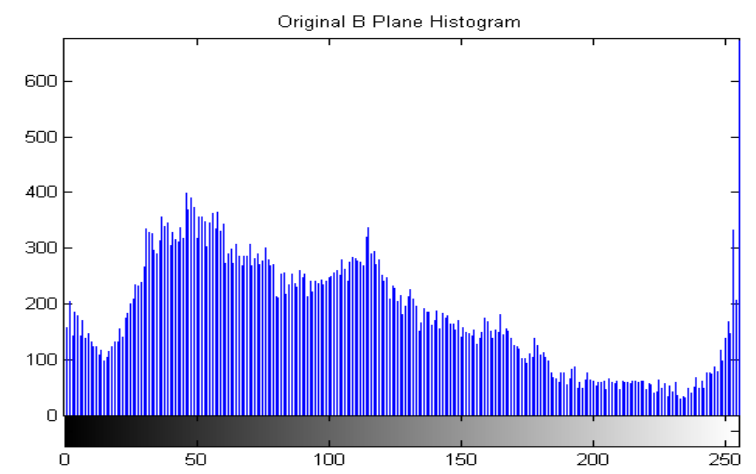

Fig. 2(E) B-component of original image .

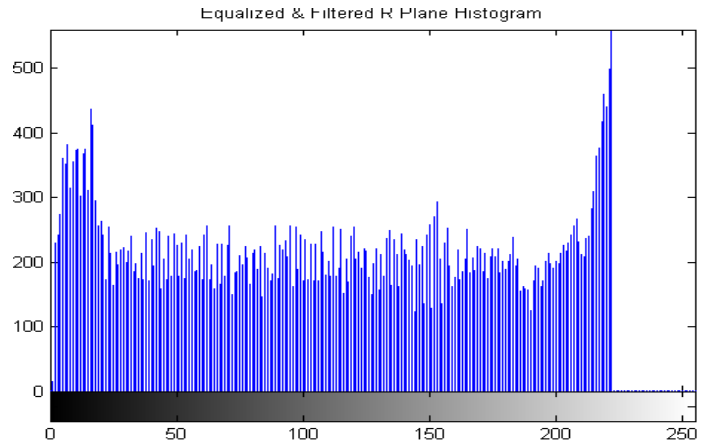

Fig. 2(B) R-component of equalized and filtered image .

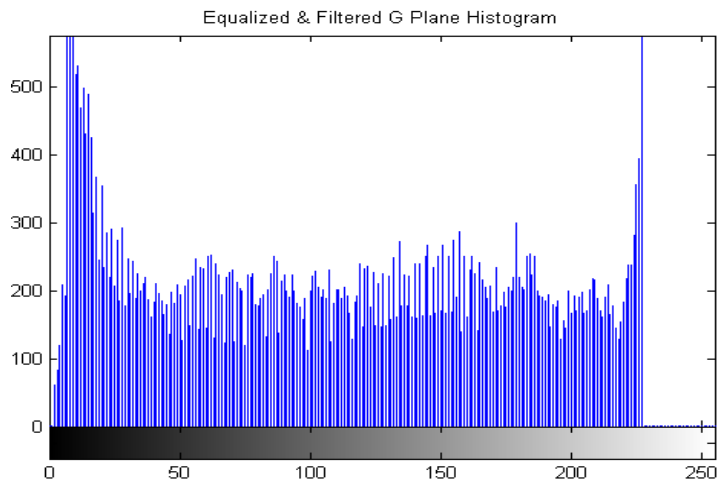

Fig. 2(D) G-component of equalized and filtered image .

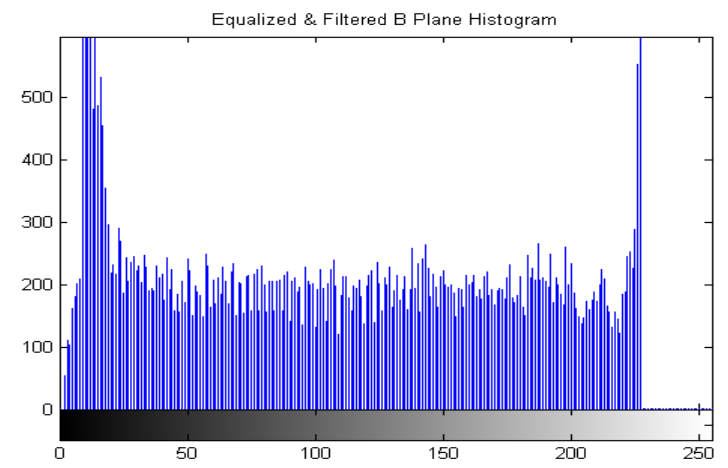

Fig. 2(F) B-component of equalized and filtered image .

Fig. 2

Fig. 2 . Histograms of Oilpill image from Remote sensing imaging regarding R, G, B color components of original and equalized and filtered by using Gaussian filter .

\subsection{Underwater Image :}

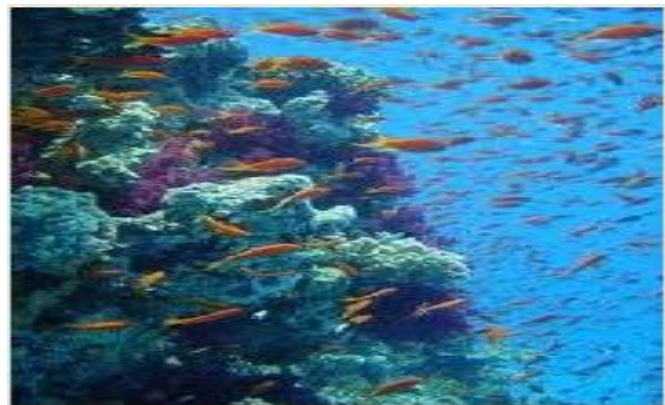

Underwater Image original image Image

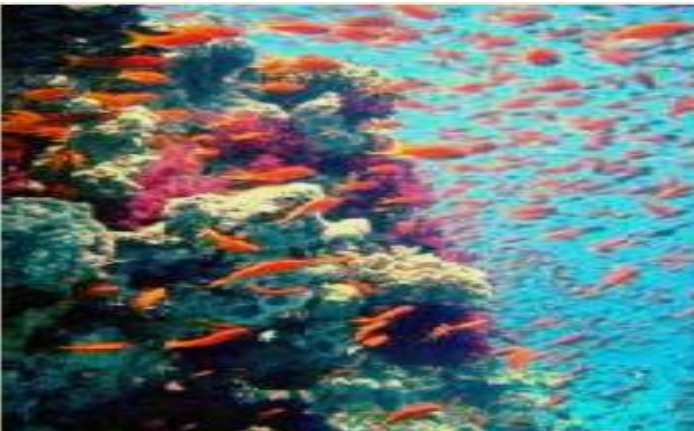

Equalized and filtered image of Underwater

Image 3 


\section{Histograms of Underwater image :}

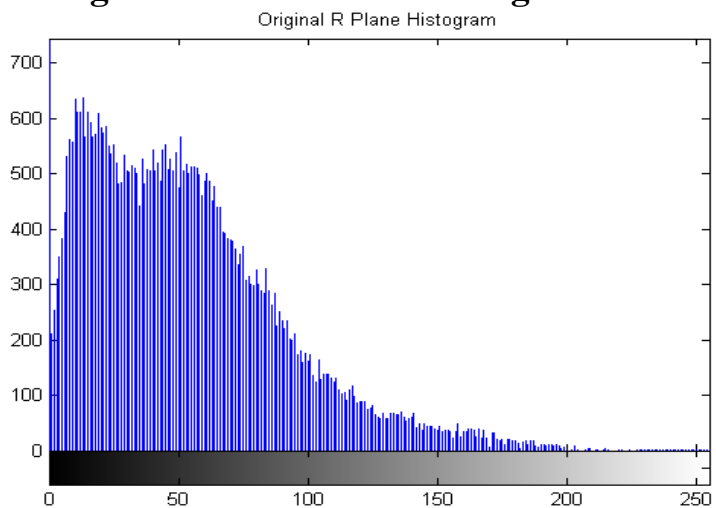

Fig. 3(A) R-component of original Underwater image .

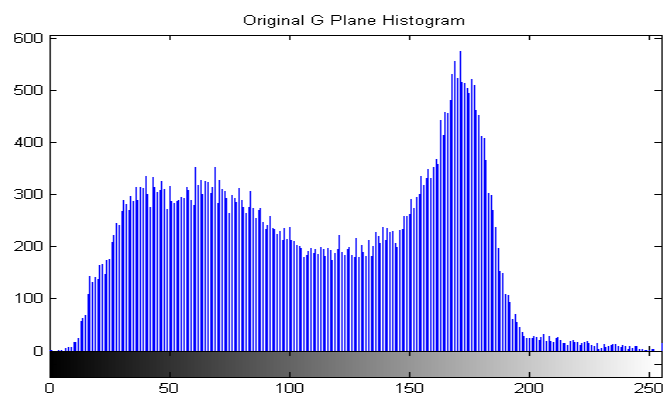

Fig. 3(C) G-component of original Underwater image .

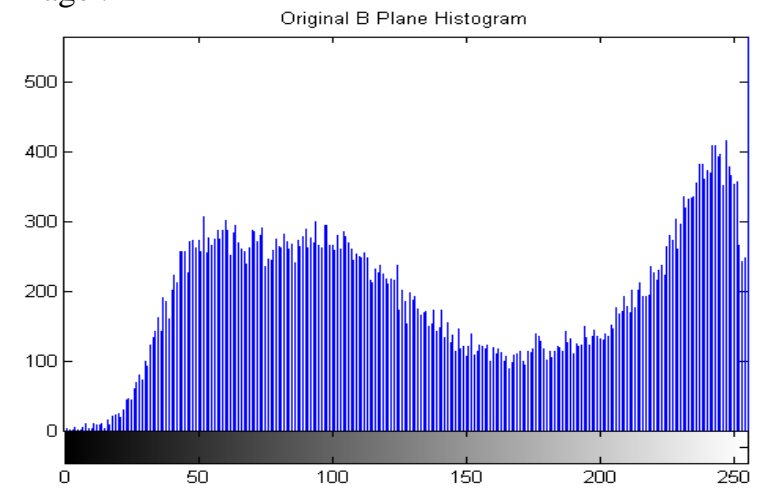

Fig. 3(E) B-component of original Underwater image .

Fig. 3

Fig. 3. Histograms of Great Barrier Reif's image regarding R , G , B color components of original and equalized and filtered by using Gaussian filter .

\subsection{NASA Img}

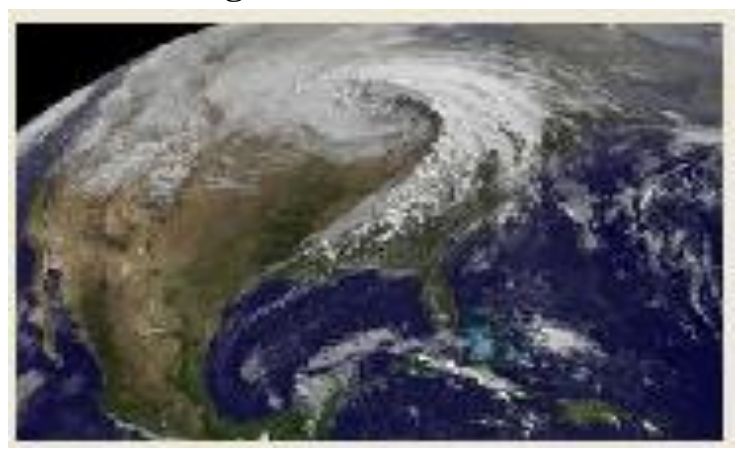

NASA original image

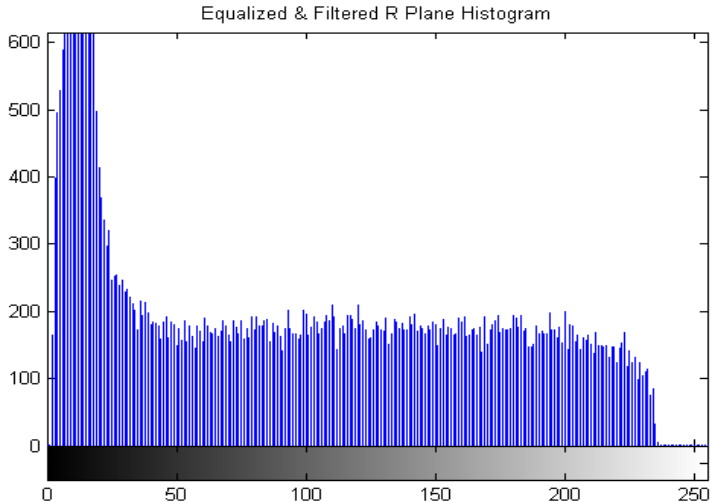

Fig. 3(B) R-component of equalized and

filtered Underwater image.

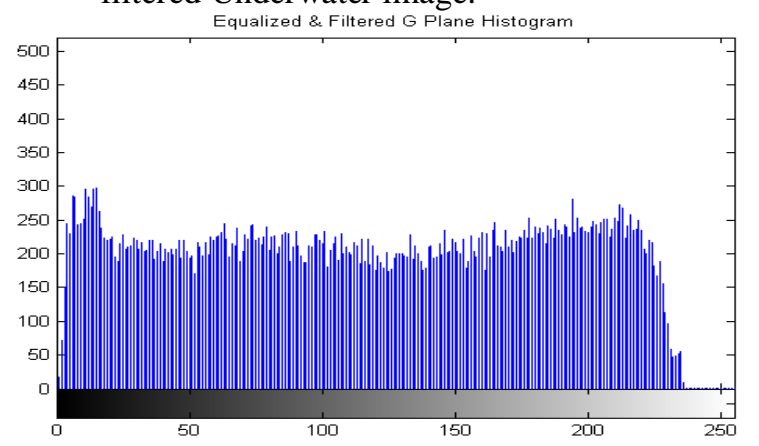

Fig. 3(D) G-component of equalized and filtered Underwater image.

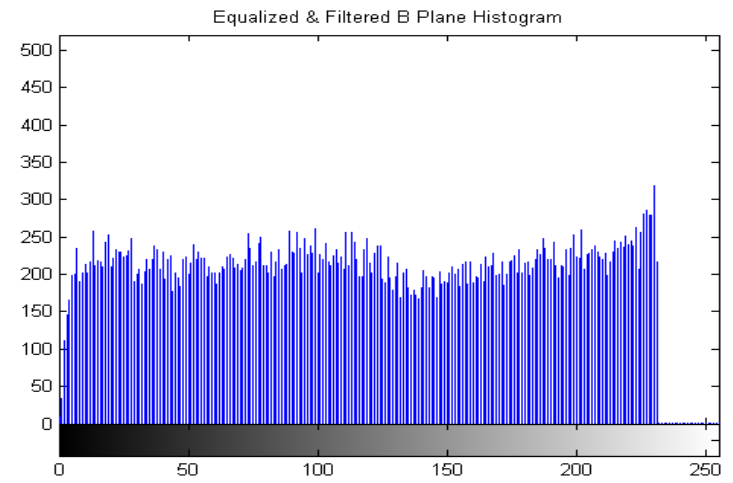

Fig. 3(F) B-component of equalized and filtered Underwater image. 
Histograms of NASA image :

Original R Plane Histogram

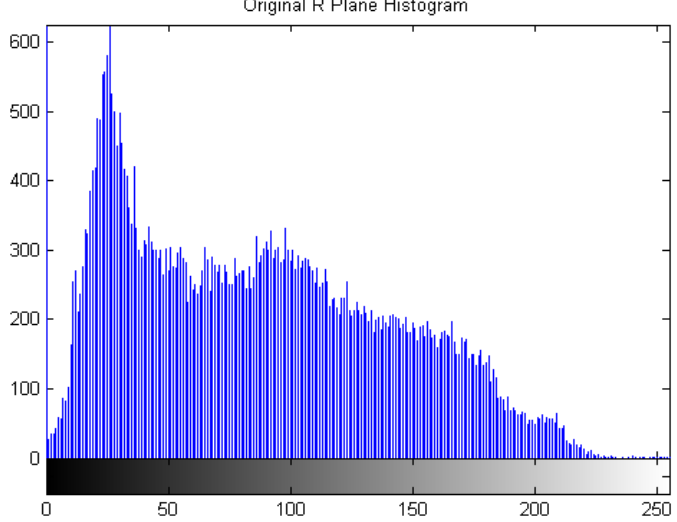

Fig. 4(A) R-component of original NASA image .

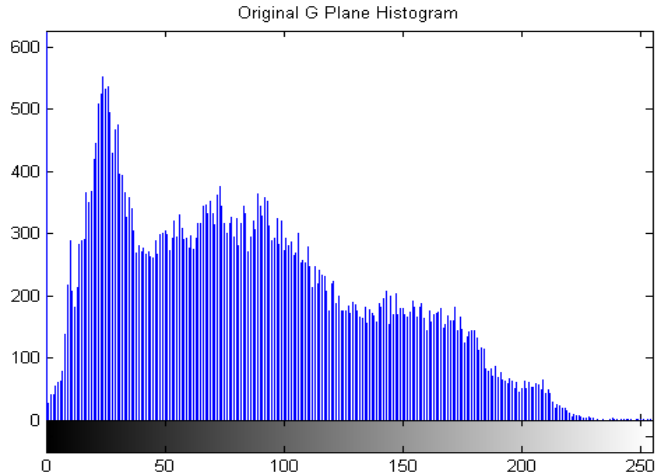

Fig. 4(C) G-component of original NASA image .

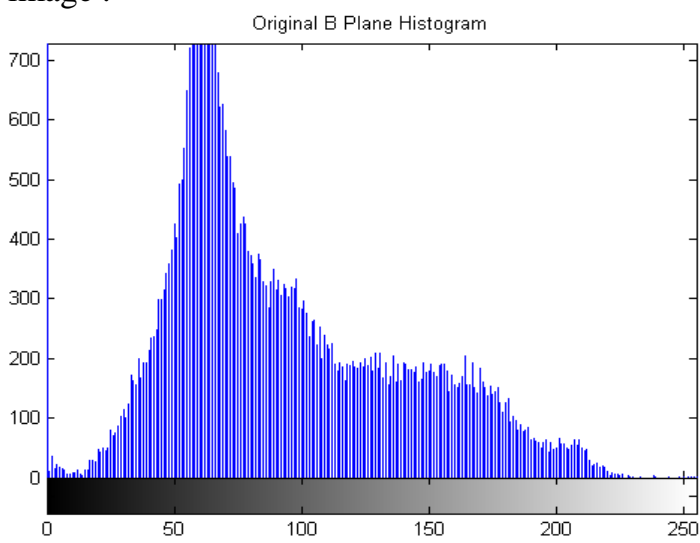

Fig. 4(E) B-component of original NASA image .

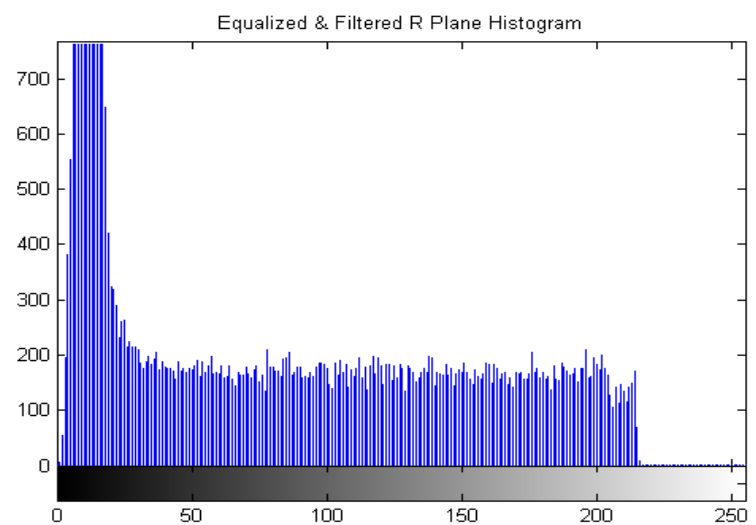

Fig. 4(B) R-component of equalized and filtered NASA image.

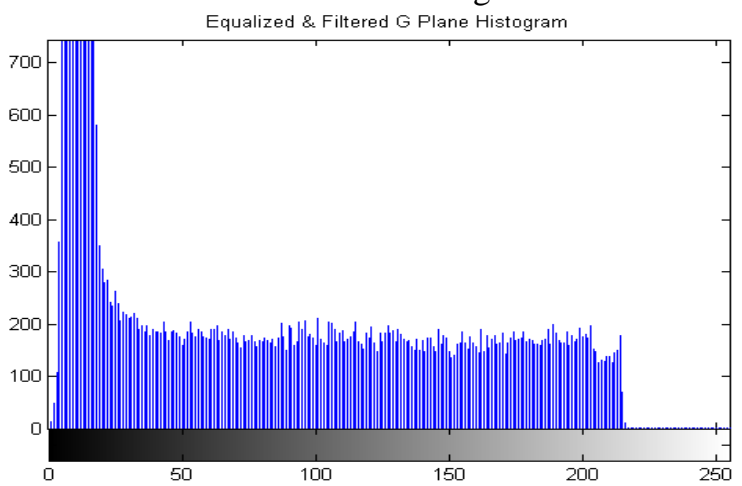

Fig. 4(D) G-component of equalized and filtered NASA image.

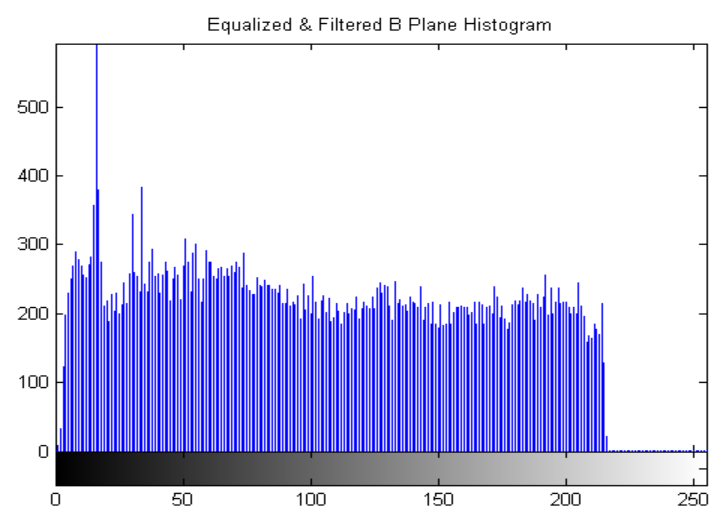

Fig. 4(F) B-component of equalized and filtered NASA image.

Fig. 4

Fig. 4 . Histograms of NASA's image regarding R , G , B color components of original and equalized and filtered by using Gaussian filter .

\section{PERFORMANCE TABLE :}

Table 1: Lungs-Vessel

\begin{tabular}{|l|l|l|l|l|}
\hline S.No & FilterUsed & MSE & PSNR & Brightness count \\
\hline 1. & Median filter & 1003.9186 & 18.1138 & 116.5979 \\
\hline 2. & Gaussian Filter & 903.4248 & 18.5719 & 116.7029 \\
\hline 3. & Average Filter & 1044.4473 & 17.9419 & 116.7051 \\
\hline 4. & Motion Filter & 1010.9364 & 18.0836 & 116.3886 \\
\hline
\end{tabular}


Table 2 : Oilpill (Remote Sensing Imaging)

\begin{tabular}{|l|l|l|l|l|}
\hline S.No & FilterUsed & MSE & PSNR & Brightnes count \\
\hline 1. & Median filter & 530.8469 & 20.8811 & 109.8058 \\
\hline 2. & Gaussian Filter & 398.4718 & 22.1268 & 109.7198 \\
\hline 3. & Average Filter & 548.9415 & 20.7355 & 109.7187 \\
\hline 4. & Motion Filter & 456.5353 & 21.5361 & 109.3989 \\
\hline
\end{tabular}

Table 3 : Great Barrier Rief (Underwater Imaging)

\begin{tabular}{|l|l|l|l|l|}
\hline S.No & FilterUsed & MSE & PSNR & Brightness count \\
\hline 1. & Median filter & 1535.861 & 16.2673 & 109.0864 \\
\hline 2. & Gaussian Filter & 1363.7329 & 16.7835 & 109.6837 \\
\hline 3. & Average Filter & 1423.5675 & 15.597 & 109.6842 \\
\hline 4. & Motion Filter & 1415.212 & 16.6226 & 109.3551 \\
\hline
\end{tabular}

Table 4 : NASA Image(Earth)

\begin{tabular}{|l|l|l|l|l|}
\hline S.No & FilterUsed & MSE & PSNR & Brightness count \\
\hline 1. & Median filter & 564.1566 & 20.6168 & 87.5739 \\
\hline 2. & Gaussian Filter & 397.5349 & 22.1371 & 87.9113 \\
\hline 3. & Average Filter & 475.7044 & 21.3574 & 87.9094 \\
\hline 4. & Motion Filter & 455.3548 & 21.5473 & 87.5898 \\
\hline
\end{tabular}

Above are some results shown on the basis of proposed technique. Here in case of Image 1, fig. 1(A), 1(C), 1(E). represents the histogram of red, green \& blue components of Original Lungs Image 1 respectively and fig. 1(B), 1(D), 1(F) shows how the intensity of red, green , blue component get equalized after the processing respectively. Similarly fig. 2(A), 2(C), 2(E) shows the histogram of Red, Green, Blue component of the original Oilpill image before processing respectively and fig. 2(B), 2(D), 2(F) shows the equalized red, green, blue component histogram of the processed image of Oilpill respectively. Similarly fig. 3(A), 3(C), 3(E) shows the red, green and blue component of the original image of Great Barrier Reef and Great Barrier Reef equalized image is shown by the fig. 3(B), 3(D), 3(F) respectively. Again from the fig. 4(A), 4(C), 4(E) we get to know about the intensity of red, green, and blue component of original NASA Image respectively and the equalized intensity of red, green, blue component of NASA image is shown by the fig. 4(B), 4(D), 4(F) respectively. From the performance table of image 1(Lungs) we came to know that by using Gaussian filter the MSE will reduced to 231.869 from 314.1955 in median filtering , 310.4368 in average filtering \& 273.4146 in motion filtering . The PSNR will be raised as 24.4784 in comparison to other filters \& last is the brightness will be preserved by the ratio 55.3679 which is also a good ratio of preserving the brightness among all the filters used here. Similarly from the performance table of Image 2(Oilpill, Remote Sensing Image) in the case of Gaussian filter MSE(1226.9706) is again reduced as compared to other filters , the value of PSNR will increased upto 17.2425 which is again greater in comparison to median, average \& motion filters Last but not the least criteria is how much brightness can be preserved so its ratio is 100.2367 which can also be acceptable as MSE and PSNR values are very good in comparison to that comes by using other filters. Again from the performance table of Image 3(Great Barrier Reef) in the case of Gaussian filter we get MSE(1042.4931) is again reduced as compared to all the three filters used here in processing , the value of PSNR will increased upto 17.9501 which is again greater in comparison to median, average \& motion filters Last but not the least criteria is how much brightness can be preserved so its ratio is 116.7488 which is also be a a good ratio in comparison to that comes by using other filters. Here comes the case with the Image 4(NASA) from the performance table in the case of Gaussian filter MSE(397.5349) is again reduced as compared to other filters, the value of PSNR will increased upto 22.1371 which is again greater in comparison to median, average \& motion filters Last but not the least criteria is how much brightness can be preserved so its ratio is 87.9113 which is also a very good ratio in comparison to that comes by using all the three filters .

\section{Conclusion}

Thus the conclusion derived from this paper is that the technique proposed here can be used to get much better contrast enhancement along with the brightness preserving with the use of Gaussian filter as 
compared with median, average, and motion filters . It provides good results with both color and gray scale images .In future this technique can be extended to be used for 3D images .

\section{Future Work and Scope}

The proposed technique can also be used for the enhancement along with the preservation of brightness and reduction of noise in 3D images and other application areas.

\section{Acknowledgement}

We would like to express our greatest gratitude to Dr. Y.K. Jain , HOD Computer Science and Engineering Dept. S.A.T.I. Vidisha (M.P) for his great support .

\section{References}

[1] R.Gonzalez and R. Woods, Digital image processing using MATLAB $5^{\text {th }} \mathrm{ed}$. Pearson, 2009

[2] Y.-T. Kim, Contrast enhancement using brightness preserving bi histogram equalization, "IEEE Trans. On Consumer Electronics, vol. 43, no. 1, pp. 1-8, Feb. 1997.

[3] David Menotti, Laurent Najman, Jacques Facon, "Multi-histogram equalization methods for contrast enhancement and brightness preserving”, "IEEE Trans. On Consumer Electronics, vol. 53, no. 3, Aug. 2007”.

[4] P. Jagatheeswari, S. Suresh Kumar and M. Rajaram, “ A novel approach for contrast enhancement based on histogram equalization followed by median filter", "ARPN Journal of Engineering and Applied Sciences, Vol. 4, No. 7, Sep. 2009.

[5] M. Karaman, L. Onural, and A. Atalar, " Design and implementation of a general purpose median filter unit in CMOS VLSI “, “ IEEE Trans." April. 1990.

[6] H.S.M.M. Hanif, M.Y. Mashor, Z. Mohamed, “ Image enhancement and segmentation using dark stretching technique for Plasmodium Falciparum for thick blood smear", “ IEEE Trans. $7^{\text {th }}$ international colloquium on signal processing and its application 2011".

[7] Virendra P.Vishwakarma, Sujata Pandey," Adaptive histogram equalization and logarithm transform with rescaled low frequency DCT coefficients for illumination normalization"," IJRTE Vol. 1, May 2009".

[8] Balvant Singh, Ravi Shankar," Analysis of Contrast Enhancement Techniques For Underwater Image”, “IJCTEE Vol 1. Issue 2."

[9] George Anastassopoulos, Ioannis Stephanakis, "Evaluation Of Histogram Enhancement Techniques Used In Conjunction With Wavelet Compression Methods For Improved Signal Processing Of Child Trauma Images" , "Seventh Australlian and New Zealand Intelligent Information System Conference, nov-2001".

[10] Ashwini Sachin Zadbuke, “ Brightness Preserving Image Enhancement Using Modified Dualistic Sub Image Histogram Equalization”, “IJSER, Vol. 3, Issue 2. Feb-2012”.

[11] H. Roseline, Aimi Salihah, A.N , M. Y. Mashor " Color Image Enhancement Technique for Acute Leukaemia Blood Cell Morphological Features “, IEEE Trans. 2010

[12] Gao Qinqing, Chen Dexin, He Ketai ," Image Enhancement Technique Based On Improved PSO Algorithm “ , IEEE Trans. 2011.

[13] Xiaodong Xie , Zaifeng Shi , Wei Guo , "An Adaptive Image Enhancement Technique Based On Image Characteristic “, IEEE Trans. 2009

[14] Zhi Yu Chen, Besma R. Abidi, David L. Page, Mongi A Abidi “ Gray- Level Grouping : An Automatic Method For Optimized Image Contrast Enhancement, The Basic Method “, IEEE Trans , 2006. 